\title{
A Generalization of Generalized Gamma Distributions
}

\author{
Mohamed Mead \\ Department of Statistics, Faculty of Commerce, Zagazig University, Egypt \\ mead9990@gmail.com \\ Mazen Nassar \\ Department of Statistics, Faculty of Commerce, Zagazig University, Egypt \\ mezo10011@gmail.com \\ Sanku Dey \\ Department of Statistics, St. Anthony's College, Shillong-793001, Meghalaya, India \\ sankud66@gmail.com
}

\begin{abstract}
For the first time, a new generalization of generalized gamma distribution called the modified generalized gamma distribution has been introduced to provide greater flexibility in modeling data from a practical viewpoint. The new distribution generalizes some recently introduced generalizations of the gamma and beta distributions . Various properties of the proposed distribution, including explicit expressions for the moments, quantiles, mode, moment generating function, mean deviation, mean residual lifetime and expression of the entropies are derived. The distribution is capable of monotonically increasing, decreasing, bathtub-shaped, and upside-down bathtub-shaped hazard rates. The maximum likelihood estimators of unknown parameters cannot be obtained in explicit forms, and they have to be obtained by solving nonlinear equations only. Two real data sets have been analyzed to show how the proposed models work in practice.
\end{abstract}

Keywords: Generalized gamma distribution, Generalized gamma function, Generalized Beta II distribution, Maximum likelihood estimation.

\section{Introduction}

In recent years, it is a common practice in the statistical distribution theory to add an extra parameter to an existing family of distribution functions. Such a technique (adding an extra parameter) is adopted to bring in more flexibility to a class of distribution functions. Besides, it can be very useful for data analysis purposes. For instance, Azzalini (1985) created the skew normal distribution by the addition of an extra parameter to the normal distribution so as to add flexibility to the normal distribution. Eugene et al. (2002) put forward the beta generated method that uses the beta distribution with parameters $\alpha$ and $\beta$ as the generator to enhance the beta generated distributions. Alzaatreh et al. (2013) proposed a new method for generating families of continuous distributions called T-X family by replacing the beta PDF with a PDF, $r(t)$, of a continuous random variable and applying a function $\mathrm{W}(\mathrm{F}(\mathrm{x}))$ that satisfies some specific conditions. Of late, Aljarrah et al. (2014) employed quantile functions to generate T-X family of distributions. For assessment of methods for generating distributions see Lee et al. (2013) \&Jones (2015).

The gamma distribution is the most popular model for analyzing skewed data. In the last few years, many generalizations of gamma and Weibull distributions are proposed. These generalizations are mainly introduced in order to extend the scope of ordinary gamma and Weibull distributions and to develop a model for failure time to suit any given 
particular situation. The generalized gamma distribution (GG) was first presented by Stacy (1962) and includes as special sub-models: the exponential, Weibull, gamma and Rayleigh distributions, among others. The GG distribution is suitable for modeling data with different types of hazard rate function: increasing, decreasing, bathtub and unimodal. The GG distribution has been used in several research areas such as engineering, hydrology and survival analysis. Agarwal and Kalla (1996) defined a new type of generalized gamma distribution by slightly modifying the form of Kobayashi's generalized gamma function (1991). Their proposed distribution may find applicability in reliability or queuing theory under the situation where the effect of all the parameters does not start in the beginning. Some of the parameters start playing their role after sometime i.e. "displaced parameter". For example, in a new machine system the corrosion problem will start after certain interval of time and similar is the case of the metal fatigue.

This paper proposes a new six parameter generalization of GG distribution, called the modified generalized gamma (MGG) distribution. It includes as special sub models such as the generalizations of gamma distribution introduced by Stacy (1962), Hoq et al. (1974), Lee and Gross (1991), Agarwal and Kalla (1996), Agarwal and Al-Saleh (2001), Kalla et al. (2001) and also the generalized beta distribution of the second kind (GB2) (McDonald,(1984)), among others. We are motivated to introduce the MGG distribution because (i) it contains a number of known lifetime sub models such as gamma, Weibull, exponential, Rayleigh, Maxwell, Chi-square, folded normal, beta type-II, Burr XII, Burr III, log-logistic distributions and so on; (ii) it is capable of modeling monotonically increasing, decreasing, bathtub-shaped, and upside-down bathtub-shaped hazard rates; (iii) it can be viewed as a suitable model for fitting the skewed data which may not be properly fitted by other common distributions and can also be used in a variety of problems in different areas such as public health, biomedical studies, and industrial reliability and survival analysis; and (iv) two real data applications show that it compares well with other competing lifetime distributions in modeling lifetime data.

This paper is organized in the following way: In Section 2, the MGG is defined and some basic distributional properties of the new model are studied. In Section 3, some wellknown and new lifetime models as members of MGG are derived. Properties of the MGG distribution are studied in Section 4 including, quantile, mode, moments, moment generating function, mean deviation, mean residual life and entropy. The maximum likelihood estimates (MLEs) of the model parameters and the corresponding observed Fisher information matrix are obtained in section 5. The potentiality of the new model is illustrated by means of application to two real data sets in Section 5. Finally, some concluding remarks are addressed in Section 6.

\section{The Modified Generalized Gamma Distribution}

The generalized gamma function which is essentially a confluent hypergeometric function has been considered by Kobayashi (1991) in the form

$$
\Gamma_{\lambda}(\alpha, k)=\int_{0}^{\infty} y^{\alpha-1}(y+k)^{-\lambda} e^{-y} d y,
$$


where $\lambda$ is a non-negative integer and $\alpha, k>0$. It is obvious that this function reduces to the ordinary gamma function $\Gamma(\alpha)$, when $\lambda=0$ that is $\Gamma_{0}(\alpha, k)=\Gamma(\alpha)$. This function is useful in many problems of diffraction theory and corrosion problems in new machines. Now we can define the following function so-called modified generalized gamma function (MGGF) with the form

$$
\Gamma_{\lambda}(\alpha, k, b)=\int_{0}^{\infty} y^{\alpha-1}(y+k)^{-\lambda} e^{-b y} d y, \quad b \geq 0, \quad \lambda \geq 0,
$$

this function can be specialized to:

i. If we take $b=1$, equation (2) reduces to the exact form of Kobayashi's function given by (1).

ii. When $b=1$ and $\lambda=0$, equation (2) becomes the standard form of gamma function in the form

$$
\Gamma(\alpha)=\int_{0}^{\infty} y^{\alpha-1} e^{-y} d y .
$$

iii. For the case of $b=0, k=1$ and $\lambda=\alpha+h$, equation (2) yields the standard form of beta function of the second type as

$$
B(\alpha, h)=\int_{0}^{\infty} y^{\alpha-1}(1+y)^{-(\alpha+h)} d y .
$$

Based on equation (2), we can define the following probability density function (pdf) $f(y ; \alpha, k, b, \lambda)=\frac{1}{\Gamma_{\lambda}(\alpha, k, b)} y^{\alpha-1}(y+k)^{-\lambda} e^{-b y}, \quad y \geq 0, \quad \alpha, k, \lambda>0, b \geq 0$,

applying the transformation $x=\theta y^{1 / \beta}$ in (5), the pdf of the MGG distribution is given by

$$
f(x ; \zeta)=\frac{\beta}{\theta \Gamma_{\lambda}(\alpha, k, b)}\left(\frac{x}{\theta}\right)^{\alpha \beta-1}\left[\left(\frac{x}{\theta}\right)^{\beta}+k\right]^{-\lambda} e^{-b(x / \theta)^{\beta}}, x \geq 0,
$$

where $\zeta=(\alpha, k, b, \lambda, \theta, \beta)^{T}, \alpha, k, \theta, \beta>0, \lambda, b \geq 0, \alpha, \beta$ are the shape parameters, $\theta, b$ are the scale parameters, $k$ is the displacement parameter and $\lambda$ is the parameter of intensity of the effect of the corresponding displacement parameter. The corresponding cumulative distribution function (cdf) is

$$
F(x ; \zeta)=\frac{\gamma_{\lambda}(\alpha, k, b, c)}{\Gamma_{\lambda}(\alpha, k, b)},
$$

where $c=(x / \theta)^{\beta}$ and $\gamma_{\lambda}(\alpha, k, b, c)$ is the lower incomplete modified generalized gamma function defined as

$$
\begin{aligned}
\gamma_{\lambda}(\alpha, k, b, c) & =\int_{0}^{c} x^{\alpha-1}(x+k)^{-\lambda} e^{-b x} d x \\
& =\Gamma_{\lambda}(\alpha, k, b)-\Gamma_{\lambda}(\alpha, k, b, c),
\end{aligned}
$$

where $\Gamma_{\lambda}(\alpha, k, b, c)$ is the upper incomplete modified generalized gamma function and given by 


$$
\Gamma_{\lambda}(\alpha, k, b, c)=\int_{c}^{\infty} x^{\alpha-1}(x+k)^{-\lambda} e^{-b x} d x
$$

The survival function and hazard rate function for MGG are, respectively, given by

$$
S(x)=\frac{\Gamma_{\lambda}(\alpha, k, b, c)}{\Gamma_{\lambda}(\alpha, k, b)}
$$

and

$$
h(x)=\frac{\beta}{\theta \Gamma_{\lambda}(\alpha, k, b, c)}\left(\frac{x}{\theta}\right)^{\alpha \beta-1}\left[\left(\frac{x}{\theta}\right)^{\beta}+k\right]^{-\lambda} e^{-b(x / \theta)^{\beta}} .
$$

Plots of the density function (6) for selected parameter values are given in Figure 1. Figure 2 displays the MGG failure rate function which can be increasing, decreasing, bathtub and upside down bathtub shaped depending on the parameter values.

(a)

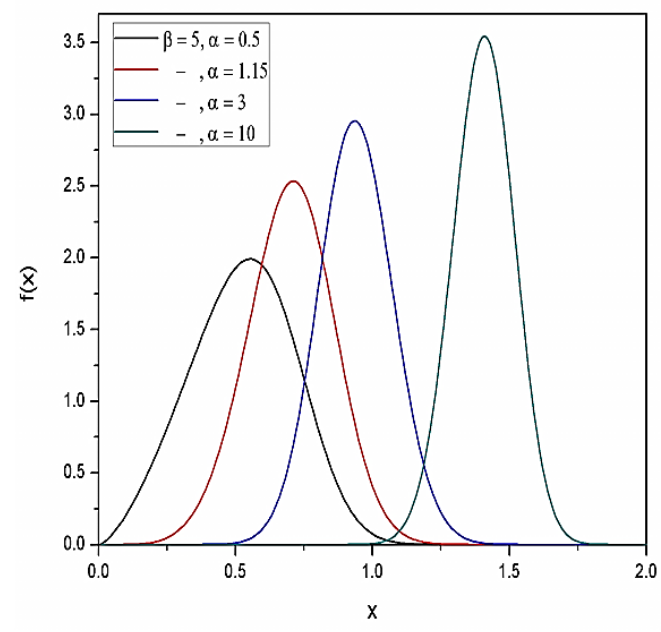

(b)

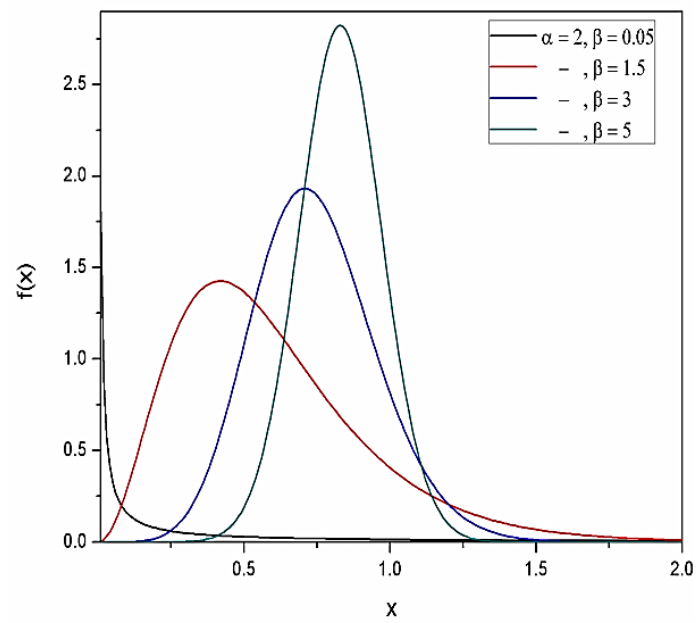

(c)

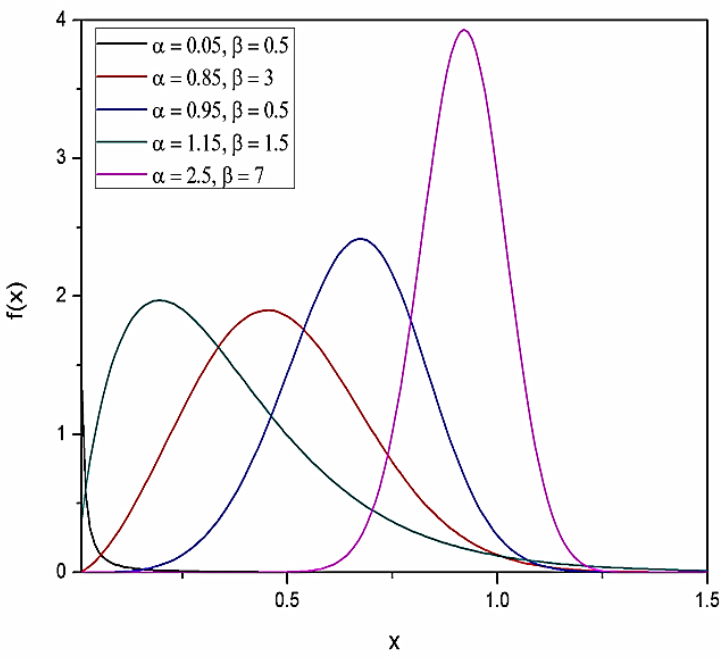


Figure 1.Density plots of MGG for $k=b=\theta=1, \lambda=5$ and (a) $\beta=5$ and various values of $\alpha$,(b) $\alpha=2$ and various values of $\beta$ and (c) various values of $\alpha$ and $\beta$.

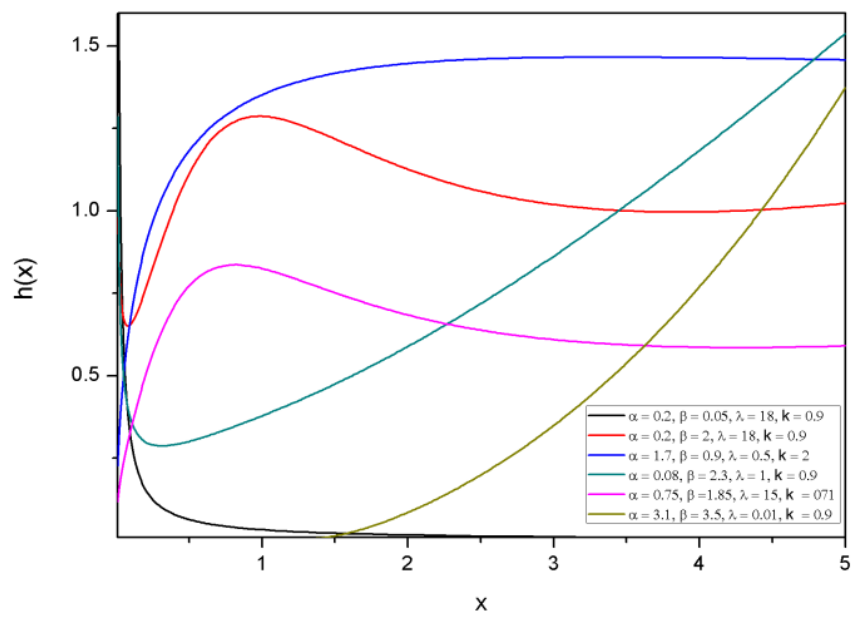

Figure 2. Hazard plots of MGG for $b=\theta=1$ and various parameter values of $\alpha, \beta, \lambda$ and $k$

\section{Special Distributions}

The MGG distribution has several distributions as special cases, which makes it distinguishable scientific importance from other distributions. In this section, we investigate the various special models of the MGG distribution.

1) When $b=1$ and $\lambda=0$, equation (6) reduces to the generalized gamma distribution given by Stacy (1962) with pdf

$$
f(x ; \beta, \alpha, \theta)=\frac{\beta}{\theta \Gamma(\alpha)}\left(\frac{x}{\theta}\right)^{\alpha \beta-1} e^{-(y / \theta)^{\beta}}, x \geq 0, \beta, \alpha, \theta>0 .
$$

In this case various special models of the MGG distribution are listed in table 1.

Table 1:Sub-models of the MGG Distribution (with $b=1$ and $\lambda=0$ )

\begin{tabular}{cccc}
\hline$\beta$ & $\theta$ & $\alpha$ & Reduced Model \\
\hline 1 & - & - & Gamma distribution \\
\hline- & - & 1 & Weibull distribution \\
\hline 2 & - & 1 & Rayleigh distribution \\
\hline 1 & - & 1 & Exponential distribution \\
\hline 2 & - & $3 / 2$ & Maxwell distribution \\
\hline 1 & 2 & $n / 2$ & Chi-square distribution \\
\hline 2 & $\sqrt{2}$ & $1 / 2$ & Folded normal distribution \\
\hline
\end{tabular}

2) In case of $b=1, \lambda=0$ and $\alpha=q / \beta$, MGG corresponds to the generalized life testing model given by Hoq et al. (1974) with pdf 


$$
f(x ; q, \beta, \theta)=\frac{\beta}{\theta \Gamma(q / \beta)}(x / \theta)^{q-1} e^{-(x / \theta)^{\beta}}, x \geq 0, q, \beta, \theta>0 .
$$

3) For $i=1,2, \ldots, n, \lambda=0, \beta=m_{i}, \alpha=b_{i}$ and $\theta=1 /\left(a_{i} p\right)$, we get the generalized gamma model defined by Lee and Gross (1991) as

$$
f\left(x_{i} ; b_{i}, m_{i}, a_{i}, p\right)=\frac{m_{i}\left(a_{i} p\right)^{-b_{i} m_{i}}}{\Gamma\left(b_{i}\right)} x_{i}^{-b_{i} m_{i}-1} e^{-\left(1 /\left(a_{i} p x_{i}\right)\right)^{m_{i}}},
$$

where $x_{i} \geq 0, b_{i}, m_{i}, a_{i}, p>0$.

4) When $b=\beta=1, \theta=1 / \delta$ and $k=\delta \gamma$, the density(6) becomes the generalized gamma model defined by Agarwal and Kalla (1996) as

$$
f(x ; \alpha, \delta, \gamma, \lambda)=\frac{\delta^{\alpha-\lambda}}{\Gamma_{\lambda}(\alpha, \delta \gamma)} x^{\alpha-1}[x+\gamma]^{-\lambda} e^{-\delta x}, x \geq 0, \alpha, \delta, \gamma, \lambda>0 .
$$

5) Kalla et al. (2001) introduced a unified type of generalized gamma model. By Setting $k=\omega \sigma / \rho, \theta^{\beta}=1 / \omega, b=1$ and $\alpha=m / \beta+1$, equation (6) yields their model with six parameters as the form

$$
f(x ; \beta, \rho, m, \sigma, \lambda, \omega)=\frac{\beta \rho^{\lambda} x^{m+\beta-1}\left[1+\rho x^{\beta} / \sigma\right]^{-\lambda} e^{-\omega x^{\beta}}}{\sigma^{\lambda} \omega^{\lambda-m / \beta-1} \Gamma_{\lambda}(m / \beta+1, \omega \sigma / \rho)},
$$

where $x \geq 0, \beta, \rho, m, \sigma, \lambda, \omega>0$. From (11), the following special cases can be derived

(i) With $\rho=\beta=1$ and $m=m-1$ we get the pdf (10).

(ii) If $\lambda=0$, the density (11) reduces to the general form of Weibull distribution with the following pdf

$$
f(x, m, \beta, \omega)=\frac{\beta \omega^{m / \beta+1} x^{m+\beta-1} e^{-\omega x^{\beta}}}{\Gamma(m / \beta+1)}, \quad m, \beta, \omega>0,
$$

which leads to the Weibull distribution with $m=0$, and the gamma distribution with $\beta=1$.

6) Agarwal and Al-Saleh (2001) used the exact function of Kobayashi (1991) to define the generalized gamma model with four parameters. The case of $b=\beta=1$ and $\theta=1 / d$ corresponds to their model with the pdf

$$
\begin{aligned}
f(x ; \alpha, k, d, \lambda)=\frac{d^{\alpha-\lambda}}{\Gamma_{\lambda}(\alpha, k)} x^{\alpha-1}[x+k / d]^{-\lambda} e^{-d x}, \\
x \geq 0, \quad \alpha, k, d, \lambda>0 .
\end{aligned}
$$

7) If we take $b=0, k=1$ and $\lambda=\alpha+h$ the pdf in (6) becomes the generalized beta distribution of the second kind (GB2) which was introduced by McDonald (1984) with the following pdf 


$$
\begin{aligned}
f(x ; \alpha, h, \beta, \theta)=\frac{\beta}{\theta B(\alpha, h)}\left(\frac{x}{\theta}\right)^{\alpha \beta-1}\left[1+\left(\frac{x}{\theta}\right)^{\beta}\right]^{-(\alpha+h)}, \\
x \geq 0, \quad \alpha, h, \theta, \beta>0,
\end{aligned}
$$

where $\alpha, \beta$ and $h$ are the shape parameters and $\theta$ is the scale parameter. The GB2 is most useful for unifying a substantial part of the size distributions literature.It contains a large number of income and loss distributions as special or limiting cases. Full details can be found in Brazauskas (2002), Kleiber and Kotz (2003).Table 2 lists various special models of the MGG distribution when $b=0$, $k=1$ and $\lambda=\alpha+h$.

Table 2:Sub-models of the MGG Distribution (with $b=0, k=1$ and $\lambda=\alpha+h$ ).

\begin{tabular}{ccccc}
\hline$\alpha$ & $\theta$ & $\beta$ & $h$ & Reduced Model \\
\hline 1 & - & - & - & Singh-Maddala (Burr XII) distribution \\
\hline- & - & - & 1 & Dagum (Burr III) distribution \\
\hline- & - & 1 & - & Beta type II distribution \\
\hline 1 & 1 & - & - & Standard Burr XII distribution \\
\hline- & 1 & - & 1 & Standard Burr III distribution \\
\hline- & 1 & 1 & - & Standard Beta type II distribution \\
\hline 1 & - & - & 1 & Lomax (or Pareto type II) distribution \\
\hline 1 & - & 1 & - & Paralogistic distribution \\
\hline 1 & - & $h$ & - & Inverse Lomax distribution \\
\hline- & - & 1 & 1 & Inverse paralogistic distribution \\
\hline- & - & $\alpha$ & - &
\end{tabular}

\section{Properties of the MGGD}

In this section, we provide some general properties of the MGG distribution including quantile function, mode, moments, mean deviation, mean residual life and mean waiting time, Rényi entropy and order statistics.

\subsection{Mode and quantile}

The $\mathrm{p}$ - thquantile function of the MGG distribution is the solution of

$$
\frac{\gamma_{\lambda}\left(\alpha, k, b,\left(x_{p} / \theta\right)^{\beta}\right)}{\Gamma_{\lambda}(\alpha, k, b)}=P,
$$

particularly, the median, denoted by $\mu^{*}$, can be obtained from (14) by substituting $p=0.5$ by solving the following

$$
\frac{\gamma_{\lambda}\left(\alpha, k, b,\left(\mu^{*} / \theta\right)^{\beta}\right)}{\Gamma_{\lambda}(\alpha, k, b)}=0.5
$$

From (6) the mode, denoted by $x_{m}$, for the MGG distribution is given by the solution of 


$$
\frac{\alpha \beta-1}{\left(x_{m} / \theta\right)^{\beta}}-\frac{\lambda \beta}{\left(\left(x_{m} / \theta\right)^{\beta}+k\right)}=b \beta .
$$

It is noted that, when $k=0$, the mode becomes

$$
x_{m}=\theta\left(\frac{(\alpha \beta-1)-\lambda \beta}{b \beta}\right)^{1 / \beta} \text {. }
$$

Also, when $b=0$ the mode reduces to

$$
x_{m}=\theta\left(\frac{k}{\lambda \beta(\alpha \beta-1)^{-1}-1}\right)^{1 / \beta} \text {. }
$$

Equations (14) and (15) are used to obtain the median and the mode for the MGG distribution. Median and mode values are reported in Table 3 for $\theta=b=k=1, \lambda=2$ and various values of $\alpha$ and $\beta$.

\section{Table 3:Median and mode of the MGG distribution}

\begin{tabular}{|c|c|c|c|}
\hline$\beta$ & $\alpha$ & Median & Mode \\
\hline 0.5 & 0.5 & 0.0081 & 0.0000 \\
\hline- & 3 & 2.3929 & 0.1716 \\
\hline- & 5 & 10.2378 & 3.0000 \\
\hline 3 & 0.5 & 0.4478 & 0.3863 \\
\hline- & 3 & 1.1565 & 1.1383 \\
\hline- & 5 & 1.4736 & 1.4657 \\
\hline 5 & 0.5 & 0.6175 & 0.6394 \\
\hline- & 3 & 1.0912 & 1.0953 \\
\hline- & 5 & 1.2619 & 1.2673 \\
\hline
\end{tabular}

From Table 3 , it is to be noted that for fixed $\theta, b, k, \lambda$ and $\beta$, the median and the mode of MGG distribution are increasing functions of $\alpha$.

\subsection{Moments, generating function and mean deviation}

From (6) it is easy to obtain the $r$-th moment about zero of MGG distribution as

$$
E\left(x^{r}\right)=\frac{\theta^{r} \Gamma_{\lambda}((r / \beta)+\alpha, k, b)}{\Gamma_{\lambda}(\alpha, k, b)} .
$$

Also, the central moments of MGG distribution can be obtained as follows

$$
E(x-\mu)^{r}=\frac{1}{\Gamma_{\lambda}(\alpha, k, b)} \sum_{j=0}^{r}\left(\begin{array}{l}
r \\
j
\end{array}\right)(-1)^{j} \mu^{j} \theta^{r-j} \Gamma_{\lambda}(\alpha+((r-j) / \beta), k, b) .
$$

where $\mu$ is the mean of the MGG distribution and can be obtained from (16) as follows 


$$
\mu=\frac{\theta \Gamma_{\lambda}((1 / \beta)+\alpha, k, b)}{\Gamma_{\lambda}(\alpha, k, b)} .
$$

using equation (17) the variance $\sigma^{2}$ can be obtained as

$$
\sigma^{2}=\frac{\theta^{2}\left[\Gamma_{\lambda}((2 / \beta)+\alpha, k, b) \Gamma_{\lambda}((1 / \beta)+\alpha, k, b)-\Gamma_{\lambda}^{2}((1 / \beta)+\alpha, k, b)\right]}{\Gamma_{\lambda}^{2}(\alpha, k, b)} .
$$

The moment generating function $M_{x}(t)$ of MGG is

$$
\begin{aligned}
M_{x}(t) & =\int_{0}^{\infty} e^{t x} f(x) d x \\
& =\sum_{j=0}^{\infty} \frac{(t \theta)^{j} \Gamma_{\lambda}[(j / \beta)+\alpha, k, b]}{j ! \Gamma_{\lambda}(\alpha, k, b)} .
\end{aligned}
$$

The mean deviation (MD)can be derived as

$$
\begin{aligned}
\mathrm{MD}=E|x-\mu| & =\int_{0}^{\infty}|x-\mu| f(x) \cdot d x \\
& =2\left\{\mu F(\mu)-\int_{0}^{\mu} x f(x) \cdot d x\right\} \\
& =\frac{2\left(\mu \gamma_{\lambda}\left(\alpha, k, b,(\mu / \theta)^{\beta}\right)-\theta \gamma_{\lambda}\left(1 / \beta+\alpha, k, b,(\mu / \theta)^{\beta}\right)\right)}{\Gamma_{\lambda}(\alpha, k, b)} .
\end{aligned}
$$

Equations (16) and (17) are used to obtain the mean, variance, skewness and kurtosis for the MGG distribution. The results are shown in Table (4) using the parameters values proposed in the previous subsection.

Table 4:Mean, variance, skewness and kurtosis of MGG distribution

\begin{tabular}{|c|c|c|c|c|c|}
\hline$\beta$ & $\alpha$ & Mean & Variance & Skewness & Kurtosis \\
\hline 0.5 & 0.5 & 0.1696 & 0.5180 & 17.1458 & 690.6574 \\
\hline- & 3 & 4.8269 & 51.7191 & 4.4978 & 41.7516 \\
\hline- & 5 & 15.5417 & 283.4986 & 3.0308 & 19.7127 \\
\hline 3 & 0.5 & 0.4755 & 0.0714 & 0.5739 & 3.0558 \\
\hline- & 3 & 1.1651 & 0.0692 & 0.1872 & 2.9401 \\
\hline- & 5 & 1.4775 & 0.0647 & 0.0950 & 2.9220 \\
\hline 5 & 0.5 & 0.6132 & 0.0497 & -0.0171 & 2.6090 \\
\hline- & 3 & 1.0892 & 0.0224 & -0.1025 & 2.8599 \\
\hline- & 5 & 1.2594 & 0.0172 & 0.0070 & 3.3573 \\
\hline
\end{tabular}

From Table 4 , it is to be noted that for fixed $\theta, b, k, \lambda$ and $\alpha$, the variance and the skewness are decreasing functions of $\beta$, while for fixed $\theta, b, k, \lambda$ and $\beta$, the mean is 
increasing function of $\alpha$. Also, it can be seen that the MGG distribution can be positively skewed, negatively skewed, platykurtic or leptokurtic.

\subsection{Mean Residual Life and Mean Waiting Time}

If the random variable $X$ follows the MGG distribution with pdf given in (6), then the mean residual life function, say $\varphi(t)$, is given by

$$
\mu(t)=E(T-t \mid T>t)=\frac{1}{S(t)} \int_{t}^{\infty} x f(x) d x-t,
$$

where $S(t)=1-F(t)$ is the survival function and

$$
\int_{t}^{\infty} x f(x) d x=\frac{\beta}{\theta \Gamma_{\lambda}(\alpha, k, b)} \int_{t}^{\infty} x(x / \theta)^{\alpha \beta-1}\left[(x / \theta)^{\beta}+k\right]^{-\lambda} e^{-b(x / \theta)^{\beta}} d x,
$$

using the transformation $y=(x / \theta)^{\beta}$, we can obtain

$$
\int_{t}^{\infty} x f(x) d x=\frac{\theta \Gamma_{\lambda}\left(\alpha+1 / \beta, k, b,(t / \theta)^{\beta}\right)}{\Gamma_{\lambda}(\alpha, k, b)},
$$

substituting (19) in (18), the mean residual function can be written as

$$
\varphi(t)=\frac{\theta \Gamma_{\lambda}\left(\alpha+1 / \beta, k, b,(t / \theta)^{\beta}\right)}{\Gamma_{\lambda}\left(\alpha, k, b,(t / \theta)^{\beta}\right)}-t .
$$

The mean waiting time of $X$, say $\bar{\varphi}(t)$, is defined by

$$
\bar{\varphi}(t)=t-\frac{m(t)}{F(t)},
$$

where $m(t)$ is the first incomplete moment and given by

$$
m(t)=\int_{0}^{t} x f(x) d x=\frac{\theta \gamma_{\lambda}\left(\alpha+1 / \beta, k, b,(t / \theta)^{\beta}\right)}{\Gamma_{\lambda}(\alpha, k, b)}
$$

now, from (20) $\bar{\varphi}(t)$ can be written as

$$
\bar{\varphi}(t)=t-\theta \frac{\gamma_{\lambda}\left(\alpha+1 / \beta, k, b,(t / \theta)^{\beta}\right)}{\gamma_{\lambda}\left(\alpha, k, b,(t / \theta)^{\beta}\right)}
$$

\subsection{Entropy}

The entropy of a random variable $X$ measures the variation of the uncertainty. The Rényi entropy, say $R E_{X}(v)$, is defined as

$$
R E_{X}(v)=\frac{1}{1-v} \ln \left(\int_{-\infty}^{\infty} f(x)^{v} d x\right), v>0, v \neq 1
$$

for the MGG distribution with PDF given by (6), we have

$$
\int_{0}^{\infty} f(x)^{v} d x=\frac{\beta^{v-1} \Gamma_{\nu \lambda}(((\alpha \beta-1) v+1) / \beta, k, b v)}{\theta^{v-1}\left[\Gamma_{\lambda}(\alpha, k, b)\right]^{v}}
$$

now, $R E_{X}(v)$ can be obtained in the following form 


$$
\begin{aligned}
R E_{X}(v)= & \ln (\theta)-\ln (\beta)+\frac{v}{v-1} \ln \left[\Gamma_{\lambda}(\alpha, k, b)\right] \\
& \left.+\frac{1}{1-v} \ln \left[\Gamma_{v \lambda}((\alpha \beta-1) v+1-\beta) / \beta, k, v b\right)\right] .
\end{aligned}
$$

It is to be noted that when $v \rightarrow 1$, the Rényi entropy converges to the Shannon entropy.

\subsection{Order statistics}

Suppose that $X_{1}, X_{2}, \ldots, X_{n}$ be a random sample of size $\mathrm{n}$, then the PDF of the $i$-th order statistic $X_{i: n}$, say $f_{i: n}(x)$ is given by

$$
f_{i: n}(x)=\frac{1}{B(i, n-i+1)} F(x)^{i-1}[1-F(x)]^{n-i} f(x),
$$

Substituting the pdf of MGG distribution given by (6) and the corresponding cdf in equation (22), we can write $f_{i: n}(x)$ as

$$
f_{i: n}(x)=\frac{\beta\left[\gamma_{\lambda}(\alpha, k, b, c)\right]^{i-1}\left[\Gamma_{\lambda}(\alpha, k, b, c)\right]^{n-i}}{\theta B(i, n-i+1)\left[\Gamma_{\lambda}(\alpha, k, b)\right]^{n}}\left(\frac{x}{\theta}\right)^{\alpha \beta-1}\left[\left(\frac{x}{\theta}\right)^{\beta}+k\right]^{-\lambda} e^{-b(x / \theta)^{\beta}}
$$

The pdf of the minimum and the maximum order statistics of MGG distribution can be obtained, respectively, from (23) as follows

$$
f_{1: n}(x)=\frac{\beta n\left[\Gamma_{\lambda}(\alpha, k, b, c)\right]^{n-1}}{\theta\left[\Gamma_{\lambda}(\alpha, k, b)\right]^{n}}\left(\frac{x}{\theta}\right)^{\alpha \beta-1}\left[\left(\frac{x}{\theta}\right)^{\beta}+k\right]^{-\lambda} e^{-b(x / \theta)^{\beta}}
$$

and

$$
f_{n: n}(x)=\frac{\beta n\left[\gamma_{\lambda}(\alpha, k, b, c)\right]^{n-1}}{\theta\left[\Gamma_{\lambda}(\alpha, k, b)\right]^{n}}\left(\frac{x}{\theta}\right)^{\alpha \beta-1}\left[\left(\frac{x}{\theta}\right)^{\beta}+k\right]^{-\lambda} e^{-b(x / \theta)^{\beta}} .
$$

The $s$-th moment of $X_{i: n}$ can be obtained as

$$
E\left(X_{i: n}^{s}\right)=\frac{\theta^{s}\left[\gamma_{\lambda}(\alpha, k, b, c)\right]^{i-1}\left[\Gamma_{\lambda}(\alpha, k, b, c)\right]^{n-i} \Gamma_{\lambda}(\alpha+s / \beta, k, b)}{B(i, n-i+1)\left[\Gamma_{\lambda}(\alpha, k, b)\right]^{n}}
$$

The joint pdf of the $i$-th and the $l$-th order statistics can be written as

$$
f_{i, l: n}(x)=C_{i, l, n} F(x)^{i-1}[1-F(y)]^{n-l}[F(y)-F(x)]^{l-i-1} f(x) f(y),
$$

where $x<y$ and $C_{i, l, n}=\frac{n !}{(i-1) !(l-i-1) !(n-l) !}$. From (24), the joint pdf of the $i$-th and the $l$-th order statistics of MGG distribution can be obtained as 


$$
\begin{aligned}
f_{i, l: n}(x) & =C_{i, l, n} \frac{\beta^{2}\left[\Gamma_{\lambda}\left(\alpha, k, b,(y / \theta)^{\beta}\right)\right]^{n-l}}{\theta^{2}\left[\Gamma_{\lambda}(\alpha, k, b)\right]^{n}} \sum_{j=0}^{l-i-1}\left(\begin{array}{c}
l-i-1 \\
j
\end{array}\right)(-1)^{j}\left[\gamma_{\lambda}\left(\alpha, k, b,(y / \theta)^{\beta}\right)\right]^{l-i-j-1} \\
& \times\left[\gamma_{\lambda}\left(\alpha, k, b,(x / \theta)^{\beta}\right)\right]^{i+j-1}\left(\frac{x}{\theta}\right)^{\alpha \beta-1}\left(\frac{y}{\theta}\right)^{\alpha \beta-1}\left[\left(\frac{x}{\theta}\right)^{\beta}+k\right]^{-\lambda}\left[\left(\frac{y}{\theta}\right)^{\beta}+k\right]^{-\lambda} \\
& \times e^{-b\left[(x / \theta)^{\beta}+(y / \theta)^{\beta}\right]}
\end{aligned}
$$

\section{Estimation of Parameters}

Here, we consider the estimation of the unknown parameters of the MGG distribution by the method of maximum likelihood. Let $x_{1}, x_{2}, \ldots \ldots, x_{n}$ be a random sample from the MGG distribution. The total $\log$-likelihood $(\ell)$ is given

$$
\ell=n \ln (\beta)-n \alpha \beta \ln (\theta)-n \ln (\delta)+(\alpha \beta-1) \sum_{i=1}^{n} \ln \left(x_{i}\right)-\lambda \sum_{i=1}^{n} \ln \left(u_{i}\right)-b \sum_{i=1}^{n} z_{i}^{\beta},
$$

where $\delta=\Gamma_{\lambda}(\alpha, k, b), z_{i}=\left(x_{i} / \theta\right)$ and $u_{i}=\left(z_{i}^{\beta}+k\right)$. The score vector

$$
\nabla \ell=\left(\frac{\partial \ell}{\partial \alpha}, \frac{\partial \ell}{\partial k}, \frac{\partial \ell}{\partial b}, \frac{\partial \ell}{\partial \lambda}, \frac{\partial \ell}{\partial \theta}, \frac{\partial \ell}{\partial \beta}\right)
$$

has the components

$$
\begin{aligned}
& \frac{\partial \ell}{\partial \alpha}=-n \beta \ln (\theta)-n \frac{\delta_{\alpha}}{\delta}+\beta \sum_{i=1}^{n} \ln \left(x_{i}\right), \frac{\partial \ell}{\partial k}=-\frac{n \delta_{k}}{\delta}-\lambda \sum_{i=1}^{n} u_{i}^{-1} \\
& \frac{\partial \ell}{\partial b}=-\frac{n \delta_{b}}{\delta}-\sum_{i=1}^{n} z_{i}^{\beta}, \frac{\partial \ell}{\partial \lambda}=-\frac{n \delta_{\lambda}}{\delta}-\sum_{i=1}^{n} \ln \left(u_{i}\right) \\
& \frac{\partial \ell}{\partial \theta}=-\frac{n \alpha \beta}{\theta}+(\beta \lambda / \theta) \sum_{i=1}^{n} u_{i}^{-1} z_{i}^{\beta}+(\beta b / \theta) \sum_{i=1}^{n} z_{i}^{\beta}
\end{aligned}
$$

and

$$
\frac{\partial \ell}{\partial \beta}=\frac{n}{\beta}-n \alpha \ln (\theta)+\alpha \sum_{i=1}^{n} \ln \left(x_{i}\right)-\lambda \sum_{i=1}^{n} u_{i}^{-1} z_{i}^{\beta} \ln \left(z_{i}\right)-b \sum_{i=1}^{n} z_{i}^{\beta} \ln \left(z_{i}\right) .
$$

From Gradshteyn and Ryzhik (2007), we can write

$$
\begin{gathered}
\Gamma_{\lambda}(\alpha, k, b)=\int_{0}^{\infty} y^{\alpha-1}(y+k)^{-\lambda} e^{-b y} d y, \quad b \geq 0, \quad \lambda \geq 0, \\
\delta_{\alpha}=\frac{\partial \delta}{\partial \alpha}=\int_{0}^{\infty} x^{\alpha-1}(x+k)^{-\lambda} e^{-b x} \ln (x) d x, \\
\delta_{k}=\frac{\partial \delta}{\partial k}=-\lambda \int_{0}^{\infty} x^{\alpha-1}(x+k)^{-(\lambda+1)} e^{-b x} d x=-\lambda \Gamma_{(\lambda+1)}(\alpha, k, b), \\
\delta_{b}=\frac{\partial \delta}{\partial b}=-\int_{0}^{\infty} x^{\alpha}(x+k)^{-\lambda} e^{-b x} d x \text { and } \delta_{\lambda}=\frac{\partial \delta}{\partial \lambda}=-\int_{0}^{\infty} x^{\alpha-1}(x+k)^{-\lambda} e^{-b x} \ln (x+k) d x
\end{gathered}
$$

The MLEs of the parameters are the solutions of the nonlinear equations $\nabla \ell=0$. The observed information matrix is given by 


$$
J(\theta)=\left[\begin{array}{cccccc}
J_{\alpha \alpha} & J_{\alpha k} & J_{\alpha b} & J_{\alpha \lambda} & J_{\alpha \theta} & J_{\alpha \beta} \\
& J_{k k} & J_{k b} & J_{k \lambda} & J_{k \theta} & J_{k \beta} \\
& & J_{b b} & J_{b \lambda} & J_{b \theta} & J_{b \beta} \\
& & & J_{\lambda \lambda} & J_{\lambda \theta} & J_{\lambda \beta} \\
& & & & J_{\theta \theta} & J_{\theta \beta} \\
& & & & & J_{\beta \beta}
\end{array}\right]
$$

whose elements are listed in Appendix.

\section{Applications}

Here, we use two real data sets to compare the fits of the MGG distribution with several other com-petitive models namely: McDonald modified Weibull (McMW) (Merovci and Elbatal (2013)), McDonald generalized Failure rate (MGLFR) (Elbatal et al. (2014)) McDonald Dagum (McDagum) (Oluyede et al. (2013)), Transmuted Weibull Lomax (TWL) (Afify et al.(2015)), Beta exponentiated Weibull (BEW) (Cordeiro et al. (2013)) and gamma exponentiated Weibull models (GEW) (Gustavo et al.(2012)) with corresponding densities(for $x \geq 0$ )

$$
\begin{aligned}
& \text { McMW: } f(x)=\frac{k\left(\theta+\lambda \beta x^{\beta-1}\right)}{B(\alpha, b)} e^{-\theta x-\lambda x^{\beta}}\left(1-e^{-\theta x-\lambda x^{\beta}}\right)^{\alpha k-1}\left[1-\left(1-e^{-\theta x-\lambda x^{\beta}}\right)^{k}\right]^{b-1}, \\
& \text { McGLFR: } f(x)=\frac{\lambda k(\theta+\beta x)}{B(\alpha, b)} e^{-\left(\theta x+\frac{\beta}{2} x^{2}\right)}\left(1-e^{-\left(\theta x+\frac{\beta}{2} x^{2}\right)}\right)^{\lambda \alpha k-1}\left[1-\left(1-e^{-\left(\theta x+\frac{\beta}{2} x^{2}\right)}\right)^{\lambda k}\right]^{b-1}, \\
& \text { McDagum :f }(x)=\frac{\lambda k \beta \theta x^{-(\theta+1)}}{B(\alpha, b)}\left(1+\lambda x^{-\theta}\right)^{-(\alpha \beta k+1)}\left[1-\left(1+\lambda x^{-\theta}\right)^{-k \beta}\right]^{b-1}, \\
& \text { TWL: } f(x)=\frac{\alpha b \beta}{\theta}[1+(x / \theta)]^{\beta b-1}\left[1-(1+(x / \theta))^{-\beta}\right]^{b-1} e^{-\alpha\left[\left(1+(x / \theta)^{\beta}\right)^{b}-1\right]} \\
& \text { BEW: } f(x)=\frac{\lambda\left[1+\lambda-2 \lambda e^{-\alpha\left[\left(1+(x / \theta)^{\beta}\right)^{b}-1\right]}\right],}{\theta B(\alpha, b)}\left(\frac{x}{\theta}\right)^{\beta-1} e^{-\lambda b\left(\frac{x}{\theta}\right)^{\beta}}\left(1-e^{-\lambda\left(\frac{x}{\theta}\right)^{\beta}}\right)^{\alpha-1}, \\
& \text { GEW: } f(x)=\frac{\beta k^{\alpha}}{\theta \Gamma(\alpha)}\left(\frac{x}{\theta}\right)^{\beta-1} e^{-\left(\frac{x}{\theta}\right)^{\beta}\left[1-e^{-\left(\frac{x}{\theta}\right)^{\beta}}\right]^{k-1}\left[-\ln \left(1-e^{-\left(\frac{x}{\theta}\right)^{\beta}}\right]\right]^{\beta-1},}
\end{aligned}
$$

where $\alpha, \beta, \lambda, \theta, b, k>0$, and $B(a, b)=\int_{0}^{1} w^{a-1}(1-w)^{b-1} d w$ is the beta function

\subsection{Application 1: Carbon Data}

The first data set is taken from Nichols and Padgett (2006) consisting of 100 observations on breaking stress of carbon fibers (in Gba). The data are: 3.7, 2.74, 2.73, 2.5, 3.6, 3.11, $3.27,2.87,1.47,3.11,4.42,2.41,3.19,3.22,1.69,3.28,3.09,1.87,3.15,4.9,3.75,2.43$, $2.95,2.97,3.39,2.96,2.53,2.67,2.93,3.22,3.39,2.81,4.2,3.33,2.55,3.31,3.31,2.85$, 2.56, 3.56, 3.15, 2.35, 2.55, 2.59,2.38, 2.81, 2.77, 2.17, 2.83, 1.92, 1.41, 3.68, 2.97, 1.36, $0.98,2.76,4.91,3.68,1.84,1.59,3.19,1.57,0.81,5.56,1.73,1.59,2,1.22,1.12,1.71$, 
2.17, $1.17,5.08,2.48,1.18,3.51,2.17,1.69,1.25,4.38,1.84,0.39,3.68,2.48,0.85,1.61$, $2.79,4.7,2.03,1.8,1.57,1.08,2.03,1.61,2.12,1.89,2.88,2.82,2.05,3.65$.

\subsection{Application 2: Repair Times Data}

The second data corresponds to 46 observations reported on active repair times (hours) for an airborne communication transceiver discussed by Alven (1964). The data are: 0.2, $0.3,0.5,0.5,0.5,0.5,0.6,0.6,0.7,0.7,0.7,0.8,0.8,1.0,1.0,1.0,1.0,1.1,1.3,1.5,1.5$, $1.5,1.5,2.0,2.0,2.2,2.5,2.7,3.0,3.0,3.3,3.3,4.0,4.0,4.5,4.7,5.0,5.4,5.4,7.0,7.5$, $8.8,9.0,10.3,22.0,24.5$.

For each model, we estimate the unknown parameters by maximum likelihood method. Tables 5 and 7 lists the MLEs (and the corresponding standard errors in parentheses) of the parameters of all the above models for both the carbon data and repair times data, respectively. We apply formal goodness- of fit tests in order to verify which distribution fits better for both data sets. The statistics we use are: $-2 \ell(\hat{\theta})$ (where $\ell(\hat{\theta})$ denotes the $\log$ likelihood function evaluated at the maximum likelihood estimates), KolmogorovSmirnov (K-S) and p-values are presented in tables 6 and 8. In general, the smaller the values of these statistics, the better the fit to the data. From these tables we observe that the MGG distribution has the lowest $-2 \ell(\hat{\theta})$, and K-S and largest p-value among all the other models, and so it could be chosen as the best model. The histogram for both carbon data and repair times data sets and their estimated pdfs for the fitted models are displayed in Figures 3(a) and 4(a)respectively. Also, the plots of the fitted MGG survival and the empirical survival functions for both carbon data and repair times data sets displayed in Figure 3(b) and Figure4(b), respectively. Therefore, the proposed model provides a better fit to these data.

Table 5: MLEs and their standard errors (in parentheses) for carbon fibers data

\begin{tabular}{ccccccc}
\hline Model & \multicolumn{7}{c}{ Estimates } \\
\cline { 2 - 7 } & $\alpha$ & $\beta$ & $\lambda$ & $\theta$ & $b$ & $k$ \\
\hline McMW & 13.5396 & 1.0064 & 0.0002 & 0.4557 & 3.1981 & 0.4999 \\
& $(31.241)$ & $(0.00665)$ & $(0.011)$ & $(0.236)$ & $(2.189)$ & $(1.080)$ \\
McGLFR & 6.48633 & 0.05391 & 0.18458 & 0.04528 & 6.56874 & 2.7366 \\
& $(12.665)$ & $(0.00002)$ & $(0.279)$ & $(0.0943)$ & $(1.222)$ & $(0.0031)$ \\
MGG & 1.37506 & 2.31925 & 0.00101 & 3.3107 & 1.92355 & $(6.2735)$ \\
& $(0.558)$ & $(0.0883)$ & $(3.414)$ & $(8.084)$ & $(8.841)$ & $(6.2735)$ \\
McDagum & 19.1107 & 0.46468 & 31.81212 & 1.3289 & 23.3537 & 0.72825 \\
& $(15.862)$ & $(0.00027)$ & $(30.815)$ & $(0.549)$ & $(23.961)$ & $(0.00042)$ \\
TWL & 1.60093 & 0.36045 & 0.8577 & 0.72681 & 4.38901 & - \\
& $(7.48)$ & $(1.434)$ & $(1.681)$ & $(5.099)$ & $(11.15)$ & \\
BEW & 0.99999 & 2.79288 & 10.9209 & 2.0646 & 0.03400 & - \\
& $(0.0012)$ & $(0.214)$ & $(6.556)$ & $(5.483)$ & $(1.051)$ & \\
GEW & 2.5151 & 2.6679 & - & 4.16461 & - & 1.52672 \\
& $(70.828)$ & $(18.651)$ & & 63.239 & & $(3.1520)$ \\
\hline
\end{tabular}


Table 6: The statistics $-2 \ell(\hat{\theta}), K-S$ and p-value for carbon fibers data

\begin{tabular}{|c|c|c|c|c|c|c|c|}
\hline \multirow{2}{*}{ Statistics } & \multicolumn{7}{|c|}{ Model } \\
\cline { 2 - 8 } & McMW & McGLFR & MGG & McDagum & TWL & BEW & GEW \\
\hline$-2 \ell(\hat{\theta})$ & 287.841 & 282.999 & $\mathbf{2 8 2 . 6 9 2}$ & 288.735 & 282.974 & 283.059 & 282.831 \\
\hline$K-S$ & 0.08703 & 0.06395 & $\mathbf{0 . 0 5 7 8 8}$ & 0.09079 & 0.06187 & 0.06048 & 0.05948 \\
\hline$p$-value & 0.43501 & 0.80801 & $\mathbf{0 . 8 9 1 0 1}$ & 0.38186 & 0.83861 & 0.85783 & 0.87104 \\
\hline
\end{tabular}

Table 7: MLEs and their standard errors (in parentheses) for repair times data

\begin{tabular}{ccccccc}
\hline Model & \multicolumn{7}{c}{ Estimates } \\
\cline { 2 - 7 } & $\alpha$ & $\beta$ & $\lambda$ & $\theta$ & $b$ & $k$ \\
\hline McMW & 0.883407 & 0.756391 & 0.000053 & 0.000053 & 1.508001 & 1.054294 \\
& $(0.19300)$ & $(0.24400)$ & $(0.00333)$ & $(0.08900)$ & $(0.70900)$ & $(0.17500)$ \\
McGLFR & 0.26848 & 0.02109 & 0.59599 & 0.01873 & 1.01245 & 2.39584 \\
& $(0.2678)$ & $(0.0001)$ & $(0.36299)$ & $(0.0135)$ & $(0.0525)$ & $(0.8438)$ \\
MGG & 13.64844 & 0.28026 & 8.07662 & 0.05747 & 3.00102 & 1.45252 \\
& $(26.324)$ & $(0.2641)$ & $(3.1350)$ & $(0.3870)$ & $(3.3430)$ & $(0.0066)$ \\
McDagum & 0.93961 & 0.92421 & 4.42377 & 1.4204 & 1.3938 & 1.11675 \\
& $(0.5040)$ & $(0.2063)$ & $(3.5050)$ & $(0.8290)$ & $(1.7180)$ & $(0.2493)$ \\
TWL & 1.6816 & 0.32074 & 0.61911 & 1.09837 & 1.45592 & - \\
& $(1.9310)$ & $(0.1540)$ & $(0.4840)$ & $(1.6610)$ & $(0.4030)$ & \\
BEW & 3.2037 & 0.88319 & 25.9070 & 3.65541 & 0.0444 & - \\
& $(1.7947)$ & $(0.0950)$ & $(7.0650)$ & $(2.3540)$ & $(0.0160)$ & \\
GEW & 3.20714 & 0.52429 & - & 4.53973 & - & 4.03723 \\
& $(2.5710)$ & $(0.3697)$ & & $(9.4820)$ & & $(6.1010)$ \\
\hline
\end{tabular}

Table 8: The statistics $-2 \ell(\hat{\theta}), K-S$ and p-value for repair times data

\begin{tabular}{|c|c|c|c|c|c|c|c|}
\hline \multirow{2}{*}{ Statistics } & \multicolumn{7}{|c|}{ Model } \\
\cline { 2 - 8 } & McMW & McGLFR & MGG & McDagum & TWL & BEW & GEW \\
\hline$-2 \ell(\hat{\theta})$ & 209.99 & 230.523 & $\mathbf{2 0 1 . 2 4 3}$ & 203.087 & 203.363 & 203.363 & 202.48 \\
\hline$K-S$ & 0.14718 & 0.19587 & $\mathbf{0 . 1 0 2 9 4}$ & 0.11351 & 0.11914 & 0.13447 & 0.10556 \\
\hline$p$-value & 0.27191 & 0.05864 & $\mathbf{0 . 7 1 4 2 9}$ & 0.59381 & 0.0 .5312 & 0.37635 & 0.68452 \\
\hline
\end{tabular}

(a)

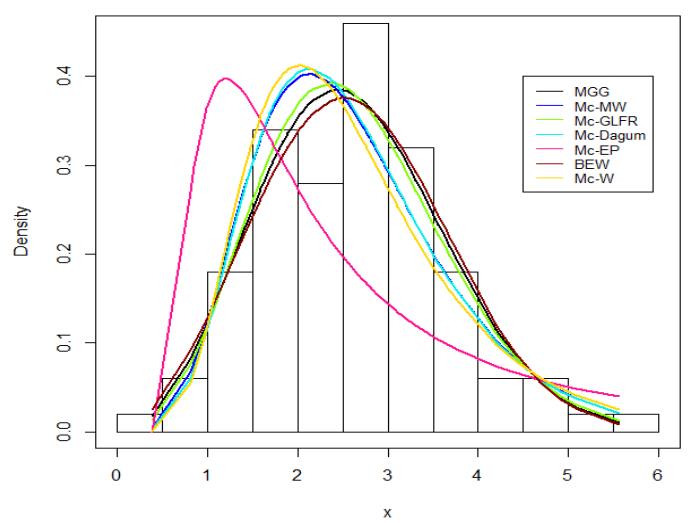

(b)

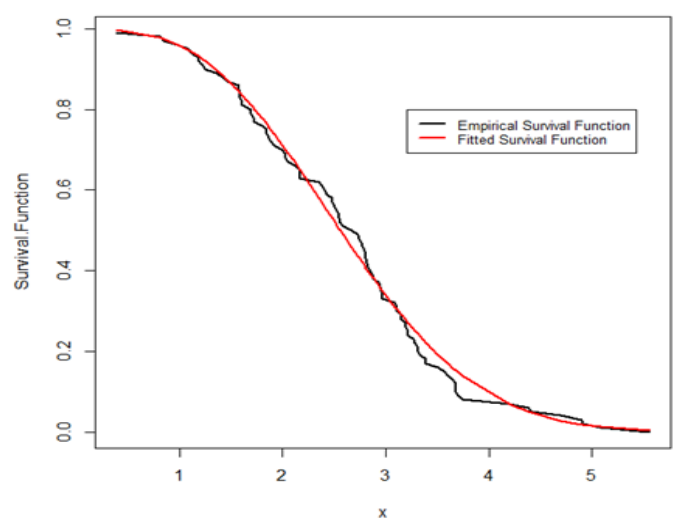

Figure 3: (a) Histogram and estimated pdfs. (b) Fitted MGG survival and empirical survival functions for repair times data 
(a)

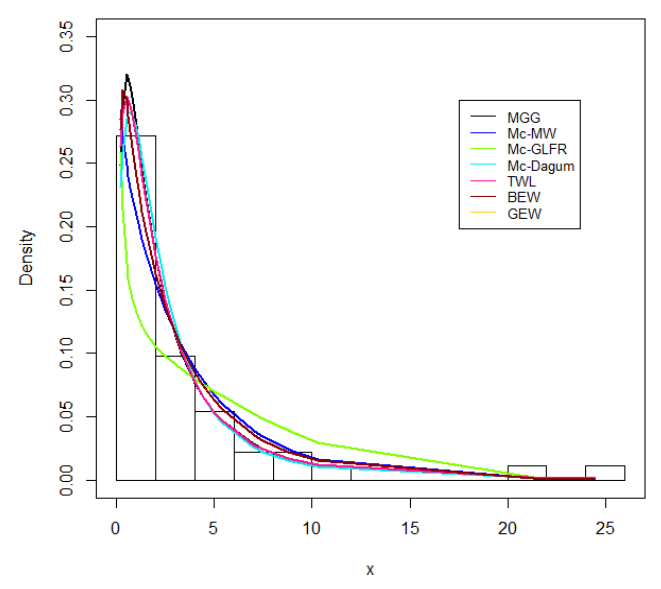

(b)

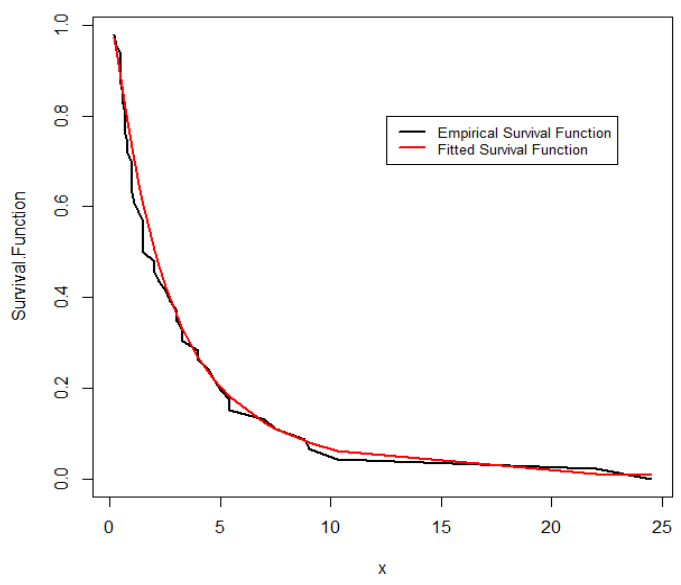

Figure 4: (a) Histogram and estimated pdfs. (b) Fitted MGG survival and empirical survival functions for carbon fibers data

\section{Conclusion}

The six-parameter MGG distribution, whose hazard function can be monotonically increasing, decreasing, bathtub and upside down bathtub-shaped depending on the parameter values, is introduced and studied. Some mathematical and statistical properties of the new model are investigated. We estimate the model parameters using maximum likelihood and determine the observed information matrix. The potentiality of the new model is illustrated by means of application to two real data sets. We hope that this model may attract wider applications, since the formulae derived are manageable using modern computer facilities.

\section{References}

1. Afify, A., Nofal, Z., Yousof, H., El Gebaly, Y. and Butt, N. (2013).The transmuted Weibull Lomax distribution. Properties and Application. Pakistan Journal of Statistics and Operation Research,11, 135-152.

2. Agarwal,S. K. and Al- Saleh, A. A.(2001). Generalized gamma type distribution and its hazard rate function. Communication in Statistics Theory-Methods,30, 309-318.

3. Agarwal, S. K. and Kalla. S. L. (1996). A generalized gamma distribution and its application in reliability. Communication in Statistics Theory-Methods, 25, 201210.

4. Aljarrah, M. A., Lee, C. and Famoye, F. (2014). On generating T-X family of distributions using quantile functions. Journal of Statistical Distributions and Applications.1, 1-17.

5. Alven, W.H., (1964).Reliability Engineering by ARINC. Prentice-hall, New Jersey. 
6. Alzaatreh, A., Lee, C. and Famoye, F. (2013). A new method for generating families of continuous distributions. Metron, 71, 63-79.

7. Azzalini, A. (1985). A class of distributions which includes the normal ones. Scandinavian journal of statistics, 12, 171-178.

8. Brazauskas, V. (2002). Fisher information matrix for the Feller-Pareto distribution. Statistics \& Probability Letters, 59, 159-167.

9. Cordeiro, G.M., Gomes, A.E., da-Silva, C.Q. and Ortega, E.M. (2013).The Beta exponentiated Weibull distribution. Journal of Statistical Computation and Simulation, 83, 114-138.

10. Elbatal, I., Merovci F. and Marzouk, W. (2014). McDonald generalized linear failure rate distribution. Pakistan Journal of Statistics and Operation Research, 10, 267-288.

11. Eugene, N. Lee,C. and Famoye, F. (2002). Beta-normal distribution and its applications. Communications in Statistics-Theory and Methods, 31, 497-512.

12. Gradshteyn, I. S. and Ryzhik, I. M.(2007). Table of integrals, series and products, Seventh Edition, Alan Jeffrey and Daniel Zwillinger (eds.), Academic Press.

13. Hoq, A., Ali, M. and Templetion (1974). Estimation of parameters of a generalized life testing models. J. Statist. Res., 9, 67-79.

14. Jones, M. (2015). On families of distributions with shape parameters. International Statistical Review, 83, 175-192.

15. Kalla, S.L. Al-Saqabi, B.N and Khajah, H.G. (2001). A unified form of gamma type distributions. Appl. Math.Comput.,118, 175-187.

16. Kleiber, C. and Kotz, S. (2003). Statistical Size Distributions in Economics and Actuarial Sciences, Hoboken, NJ: Wiley-Inter Science.

17. Kobayashi, K. (1991). On generalized gamma functions occurring in diffraction Theory. Journal Physical Society of Japan, 60, 1501-1512.

18. Lee, M. and Gross, A. (1991). Lifetime distributions under unknown environment. Journal. Statist. Planning and Inference, 29, 137-143.

19. Lee, C., Famoye, F. and Alzaatreh, A. (2013). Methods for generating families of univariate continuous distributions in the recent decades. Wiley Interdisciplinary Reviews: Computational Statistics, 5, 219-238.

20. McDonald, J.B. (1984). Some generalized functions for the size distribution of income. Econometrica, 52, 647- 663.

21. Merovci, F. and Elbatal, I. (2013). The McDonald modified Weibull distribution: properties and applications. arXiv preprint arXiv, 1309- 2961.

22. Nichols, M.D. and Padgett, W.J. (2006).A Bootstrap control chart for Weibull percentiles. Quality and Reliability Engineering International, 22, 141-151.

23. Oluyede, B. O. and Rajasooriya, S. (2013). The Mc-Dagum distribution and its statistical properties with applications. Asian Journal Mathematics and Applications, 44, 1-16. 
24. Pinho, L., Cordeiro, G. and Nobre, J. (2012). The gamma-exponentiated Weibull distribution. Journal of Statistical Theory and Applications, 11, 379-395.

25. Stacy, E. W. (1962). A generalization of the gamma distribution. Annals of Mathematical Statistics, 33, 1187-1192.

\section{Appendix}

$$
\begin{aligned}
& J_{\alpha \alpha}=-\frac{\partial^{2} \ell}{\partial \alpha^{2}}=\frac{n\left(\delta \delta_{\alpha \alpha}-\delta_{\alpha}^{2}\right)}{\delta^{2}}, J_{\alpha k}=-\frac{\partial^{2} \ell}{\partial \alpha \partial k}=\frac{n\left(\delta \delta_{\alpha k}-\delta_{\alpha} \delta_{k}\right)}{\delta^{2}}, \\
& J_{\alpha b}=-\frac{\partial^{2} \ell}{\partial \alpha \partial b}=\frac{n\left(\delta \delta_{\alpha b}-\delta_{\alpha} \delta_{b}\right)}{\delta^{2}}, J_{\alpha \lambda}=-\frac{\partial^{2} \ell}{\partial \alpha \partial \lambda}=\frac{n\left(\delta \delta_{\alpha \lambda}-\delta_{\alpha} \delta_{\lambda}\right)}{\delta^{2}}, \\
& J_{\alpha \theta}=-\frac{\partial^{2} \ell}{\partial \alpha \partial \theta}=(n \beta / \theta), J_{\alpha \beta}=-\frac{\partial^{2} \ell}{\partial \alpha \partial \beta}=n \ell n(\theta)-\sum_{i=1}^{n} \ln \left(x_{i}\right), \\
& J_{k k}=-\frac{\partial^{2} \ell}{\partial k^{2}}=\frac{n\left(\delta \delta_{k k}-\delta_{k}^{2}\right)}{\delta^{2}}-\lambda \sum_{i=1}^{n} u_{i}^{-2}, J_{k b}=-\frac{\partial^{2} \ell}{\partial k \partial b}=\frac{n\left(\delta \delta_{k b}-\delta_{k} \delta_{b}\right)}{\delta^{2}}, \\
& J_{k \lambda}=-\frac{\partial^{2} \ell}{\partial k \partial \lambda}=\frac{n\left(\delta \delta_{k \lambda}-\delta_{k} \delta_{\lambda}\right)}{\delta^{2}}+\sum_{i=1}^{n} u_{i}^{-1}, J_{k \theta}=-\frac{\partial^{2} \ell}{\partial k \partial \theta}=(\lambda \beta / \theta) \sum_{i=1}^{n} u_{i}^{-2} z_{i}^{\beta}, \\
& J_{k \beta}=-\frac{\partial^{2} \ell}{\partial k \partial \beta}=-\sum_{i=1}^{n} u_{i}^{-2} z_{i}^{\beta} \ln \left(z_{i}\right), J_{b b}=-\frac{\partial^{2} \ell}{\partial b^{2}}=\frac{n\left(\delta \delta_{b b}-\delta_{b}^{2}\right)}{\delta^{2}}, \\
& J_{b \lambda}=-\frac{\partial^{2} \ell}{\partial b \partial \lambda}=\frac{n\left(\delta \delta_{b \lambda}-\delta_{b} \delta_{\lambda}\right)}{\delta^{2}}, J_{b \theta}=-\frac{\partial^{2} \ell}{\partial b \partial \theta}=-(\beta / \theta) \sum_{i=1}^{n} z_{i}^{\beta}, \\
& J_{\theta \beta}=-\frac{\partial^{2} \ell}{\partial \theta \partial \beta}=n \frac{\alpha}{\theta}+(\lambda / \theta) \sum_{i=1}^{n} u_{i}^{-1} z_{i}^{\beta}\left(\beta k u_{i}^{-1} \ln \left(z_{i}\right)+1\right)+(b / \theta) \sum_{i=1}^{n} z_{i}^{\beta}\left(\beta \ln \left(z_{i}\right)+1\right) \\
& J_{b \beta}=-\frac{\partial^{2} \ell}{\partial b \partial \beta}=\sum_{i=1}^{n} z_{i}^{\beta} \ln \left(z_{i}\right), J_{\lambda \lambda}=-\frac{\partial^{2} \ell}{\partial \lambda^{2}}=\frac{n\left(\delta \delta_{\lambda \lambda}-\delta_{\lambda}^{2}\right)}{\delta^{2}}, \\
& J_{\lambda \theta}=-\frac{\partial^{2} \ell}{\partial \lambda \partial \theta}=-(\beta / \theta) \sum_{i=1}^{n} u_{i}^{-1} z_{i}^{\beta}, J_{\lambda \beta}=-\frac{\partial^{2} \ell}{\partial \lambda \partial \beta}=\sum_{i=1}^{n} u_{i}^{-1} z_{i}^{\beta} \ln \left(z_{i}\right), \\
& J^{2}+\left(\beta \lambda / \theta^{2}\right) \sum_{i}^{n} z_{i}^{\beta} u_{i}^{-1}\left(\beta k u_{i}^{-1}+1\right)+\left(\beta b(\beta+1) / \theta^{2}\right) \sum_{i}^{\beta},
\end{aligned}
$$

and

$$
J_{\beta \beta}=-\frac{\partial^{2} \ell}{\partial \beta^{2}}=\frac{n}{\beta^{2}}+k \lambda \sum_{i=1}^{n} z_{i}^{\beta} u_{i}^{-2} \ln ^{2}\left(z_{i}\right)+b \sum_{i=1}^{n} z_{i}^{\beta} \ln ^{2}\left(z_{i}\right) .
$$

where

$$
\delta_{\alpha \alpha}=\frac{\partial^{2} \delta}{\partial \alpha^{2}}=\int_{0}^{\infty} x^{\alpha-1}(x+k)^{-\lambda} e^{-b x} \ln ^{2}(x) d x,
$$




$$
\begin{aligned}
& \delta_{\alpha k}=\frac{\partial^{2} \delta}{\partial \alpha \partial k}=-\lambda \int_{0}^{\infty} x^{\alpha-1}(x+k)^{-(\lambda+1)} e^{-b x} \ln (x) d x, \\
& \delta_{\alpha b}=\frac{\partial^{2} \delta}{\partial \alpha \partial b}=-\int_{0}^{\infty} x^{\alpha}(x+k)^{-\lambda} e^{-b x} \ln (x) d x, \\
& \delta_{\alpha \lambda}=\frac{\partial^{2} \delta}{\partial \alpha \partial \lambda}=-\int_{0}^{\infty} x^{\alpha-1}(x+k)^{-\lambda} e^{-b x} \ln (x) \ln (x+k) d x, \\
& \delta_{k k}=\frac{\partial^{2} \delta}{\partial k^{2}}=\lambda(\lambda+1) \int_{0}^{\infty} x^{\alpha-1}(x+k)^{-(\lambda+2)} e^{-b x} d x=\lambda(\lambda+1) \Gamma_{(\lambda+2)}(\alpha, k, b), \\
& \delta_{k b}=\frac{\partial^{2} \delta}{\partial k \partial b}=\lambda \int_{0}^{\infty} x^{\alpha}(x+k)^{-(\lambda+1)} e^{-b x} d x, \\
& \delta_{k \lambda}=\frac{\partial^{2} \delta}{\partial k \partial \lambda}=-\int_{0}^{\infty} x^{\alpha-1}(x+k)^{-(\lambda+1)} e^{-b x}[1-\lambda \ln (x+k)] d x, \\
& \delta_{b b}=\frac{\partial^{2} \delta}{\partial b^{2}}=\int_{0}^{\infty} x^{\alpha+1}(x+k)^{-\lambda} e^{-b x} d x, \delta_{b \lambda}=\frac{\partial^{2} \delta}{\partial b \partial \lambda}=\int_{0}^{\infty} x^{\alpha}(x+k)^{-\lambda} e^{-b x} \ln (x+k) d x
\end{aligned}
$$

and

$$
\delta_{\lambda \lambda}=\frac{\partial^{2} \delta}{\partial \lambda^{2}}=\int_{0}^{\infty} x^{\alpha-1}(x+k)^{-\lambda} e^{-b x} \ln ^{2}(x+k) d x .
$$

